\title{
MYELIN FORMATION IN ROTATION-MEDIATED AGGREGATING CELL CULTURES: IMMUNOCYTOCHEMICAL, ELECTRON MICROSCOPIC, AND BIOCHEMICAL OBSERVATIONS ${ }^{1}$
}

\author{
BRUCE D. TRAPP, ${ }^{*}, 2$ HENRY DEF. WEBSTER, ${ }^{*}$ DAVID JOHNSON, \\ STEVEN R. COHEN,§ AND MARGARET R. MURRAY* \\ ${ }^{*}$ Laboratory of Neuropathology and Neuroanatomical Sciences, $\ddagger$ Developmental and Metabolic Neurology Branch, National \\ Institute of Neurological and Communicative Disorders and Stroke, National Institutes of Health, Bethesda, Maryland 20205 \\ and §Department of Neurology, The Johns Hopkins University School of Medicine, Baltimore, Maryland 21205
}

Received November 16, 1981; Revised February 15, 1982; Accepted February 16, 1982

\begin{abstract}
Myelination was studied in aggregating cell cultures derived from mechanically dissociated 15- to 16-day fetal rat brains. Myelin basic protein (MBP) and myelin-associated glycoprotein (MAG) were localized immunocytochemically in 20 - $\mu \mathrm{m}$-thick Vibratome and 1- $\mu \mathrm{m}$-thick Epon sections at 15, 20, 25 , and 30 days in vitro. The occurrence of these proteins was correlated with the ultrastructural appearance of oligodendrocytes and myelin sheaths and with biochemical levels of MBP, MAG, and the myelin-related enzyme, $2^{\prime}, 3^{\prime}$-cyclic nucleotide $3^{\prime}$-phosphodiesterase (CNP). MBP appeared in ultrastructurally immature oligodendrocyte cytoplasm at 15 days in vitro. As oligodendrocytes developed a more differentiated fine structure, MBP and MAG antisera stained oligodendrocyte processes and myelin sheaths. Immunostaining in Vibratome sections demonstrated that MBP was detectable in oligodendrocytes and myelin prior to MAG. At 25 days in vitro, all Vibratome sections contained MBP- and MAG-stained oligodendrocytes and myelin sheaths.

Radioimmunoassays for MBP and MAG and enzyme assays for CNP in whole homogenates of the aggregates revealed that each of these components increased with the progression of myelination. However, MBP only reached $8 \%$ of the level in adult rat brain, while MAG and CNP increased to more than half of the adult level. The protein composition of myelin purified from 30-day aggregates resembled that of myelin purified from immature rat brain.
\end{abstract}

Aggregating cell cultures of fetal rat brain are a useful in vitro model for the multidisciplinary investigation of CNS development (Matthieu et al., 1980; Trapp and Richelson, 1980; Trapp et al., 1981b). The cells within the aggregates interact by cell-cell contracts in three dimensions and are independent of contact with the surface of their culture vessel. Each culture flask contains several thousand aggregates (8 to $10 \mathrm{mg}$ of tissue protein). The yield of tissue is sufficient for standard biochemical analysis of whole aggregates and subcellular fractions isolated from aggregate homogenates. Fifty to 100 aggregates can be harvested sequentially for morphological examination or, at individual time points, aggregates from the same

\footnotetext{
'We wish to thank Raymond Rusten of the Infectious Diseases Branch at the National Institutes of Health for printing the micrographs and Judy Hertler for typing the manuscript.

${ }^{2}$ To whom correspondence should be addressed at his present address: Laboratory of Molecular Genetics, National Institute of Neurological and Communicative Disorders and Stroke, Building 36, Room 4A-01, National Institutes of Health, Bethesda, MD 20205.
}

flask can be used for both morphological and biochemical analysis. This allows correlation of morphological and biochemical results from tissue maintained in the same culture vessel.

The morphological and biochemical differentiation thus far observed in aggregates resembles that found in normal brain (Seeds, 1973; Garber, 1977; Trapp et al., 1979a, 1981b; Matthieu et al., 1980; Trapp and Richelson, 1980). Aggregates develop from morphologically undifferentiated neuroepithelial cells to a population of morphologically mature neurons, astrocytes, and oligodendrocytes (Trapp et al., 1979a). Synaptic contacts and myelinated axons appear as the cells differentiate. At least four putative neurotransmitters are synthesized in aggregates (Honegger and Richelson, 1979), and neurotransmitter-metabolizing enzymes can attain specific activities which are similar to those found in adult rat brain (Honegger and Richelson, 1976, 1977). Myelin-specific (or -associated) proteins, lipids, and enzymes increase with in vitro age in a developmental pattern similar to that in vivo (Schmidt, 1975; Sheppard et al., 1978; Mat- 
thieu et al., 1978, 1979, 1980; Bourre et al., 1979), and myelin can be isolated in sufficient quantities for biochemical analysis (Matthieu et al., 1978).

In this report, we describe immunocytochemical, electron microscopic, and biochemical studies of myelin formation in aggregating cultures. Our results demonstrate the advantages of aggregating cell cultures for the multidisciplinary investigation of myelin formation and show that early stages of myelinogenesis in aggregating cultures mimic those found in normal brain. A preliminary report of this work has already appeared (Trapp et al., 1979c).

\section{Materials and Methods}

\section{Reaggregating cultures}

Whole brains from 15- to 16-day Sprague-Dawley rat embryos were dissociated mechanically into single cells and reaggregated under constant rotation as previously described (Trapp and Richelson, 1980). Three flasks of aggregates were harvested for combined electron microscopic, immunocytochemical, and biochemical analysis at $15,20,25$, and 30 days in vitro. Twenty additional flasks were harvested at 30 days in vitro for characterization of isolated myelin.

\section{Morphological studies}

Approximately $10 \%$ of the aggregates from each of the three flasks harvested at $15,20,25$, and 30 days in vitro were pooled for electron microscopic and immunocytochemical studies. At each time point, half of the pooled aggregates were used for electron microscopic analysis and for the immunocytochemical localization of myelin basic protein (MBP) and myelin-associated glycoprotein (MAG) in 1- $\mu \mathrm{m}$-thick Epon sections. The remaining aggregates were used to localize MBP and MAG in $20-\mu \mathrm{m}$ thick Vibratome sections.

\section{Electron microscopy}

Aggregates were fixed by immersion in an aldehyde solution containing $1.5 \%$ glutaraldehyde and $0.5 \%$ paraformaldehyde in $0.08 \mathrm{~m}$ phosphate buffer. Following $2 \mathrm{hr}$ of fixation, the aggregates were postfixed in $1 \%$ osmium tetroxide for $1 \mathrm{hr}$, dehydrated in ethanol, and embedded in Epon. Thin sections were stained with uranyl acetate and lead citrate and examined in a Philips 400 electron microscope.

\section{Immunocytochemistry}

Vibratome sections. Aggregates were fixed by immersion in a solution containing $76 \mathrm{ml}$ of saturated $\mathrm{HgCl}_{2}$ and $20 \mathrm{ml}$ of $37 \%(\mathrm{v} / \mathrm{v})$ formaldehyde. Following $2 \mathrm{hr}$ of fixation, the aggregates were rinsed three times with 0.5 $\mathrm{M}$ Tris buffer, embedded in $10 \%$ gelatin, and sectioned at a thickness of $20 \mu \mathrm{m}$ on a vibrating microtome. Alternate sections were stained immunocytochemically with MBP or MAG antisera by the peroxidase-antiperoxidase (PAP) method as previously described (Sternberger et al., 1978b, 1979; Trapp et al., 1979b, 1981b). Following the immunostaining steps, the sections were infiltrated with glycerol, mounted on glass slides, and examined with a Zeiss differential interference contrast microscope.
The percentage of $20-\mu$ m-thick Vibratome sections containing at least one MBP- or MAG-stained oligodendrocyte and/or myelin sheath was determined at 15, 20, 25 , and 30 days in vitro. Aggregate sections having a diameter within $1 \mathrm{SD}$ of the mean were analyzed (Trapp et al., 1979a). This assured that comparable areas of each aggregate were studied at each time point. The number of stained cells and myelin sheaths in each section was not counted.

Epon sections. One-micrometer-thick Epon sections from the tissue used for electron microscopic studies were cut with glass knives, mounted on glass slides, and placed in a 60 to $80^{\circ} \mathrm{C}$ oven for 24 to $48 \mathrm{hr}$. The sections were encircled with a diamond scribe and pretreated with sodium ethoxide and hydrogen peroxide prior to immunostaining as previously described (Trapp et al., 1981a). The sections were stained immunocytochemically by the PAP method (Sternberger et al., 1970). Following the immunostaining steps, the sections were mounted in Harleco synthetic resin and examined with a Zeiss brightfield microscope.

The MBP and MAG antisera have been well characterized (Cohen et al., 1975; Quarles et al., 1981). The specificity of MBP and MAG immunostaining was tested by incubating $1-\mu \mathrm{m}$ Epon and $20-\mu \mathrm{m}$ Vibratome sections with preimmune serum or MBP and MAG antisera which were absorbed with purified MBP and MAG.

\section{Biochemistry}

Levels of MBP, MAG, and $2^{\prime}, 3^{\prime}$-cyclic nucleotide $3^{\prime}$ phosphodiesterase (CNP) were determined in homogenates of aggregates. Three separate flasks were measured in duplicate at $15,20,25$, and 30 days in culture. MBP was measured by radioimmunoassay (RIA) by the method of Cohen et al. (1975). MAG was measured by RIA by the method of Johnson et al. (1980). CNP was determined by the method of Kurihara and Tsukada (1967). Protein was determined by the method of Lowry et al. (1951).

Myelin was isolated from 30-day-old aggregates and brains of adult and 14-day-old rats by the method of Norton and Poduslo (1973). Myelin proteins were partially delipidated and then solubilized with SDS as previously described (Quarles and Everly, 1977) and separated on $12.5 \%$ polyacrylamide slab gels (Laemmli, 1970). Samples of $20 \mu \mathrm{g}$ of myelin proteins were applied to each lane. The gels were stained with $0.25 \%$ Coomassie blue.

\section{Results}

Immunocytochemistry and electron microscopy. In aggregating cell cultures of fetal rat brain, MBP was found in oligodendrocyte cytoplasm when only a few myelin sheaths had developed (Fig. 1). MBP-immunostained cells were usually round or oval in shape and contained a thin rim of reaction product around their nuclei. A few cells had stained processes extending from their perikarya, and these processes occasionally surrounded axons or were continuous with myelin sheaths. Thirty-three percent of the MBP-stained Vibratome sections from 15day aggregates contained at least one stained oligodendrocyte, but only $10 \%$ contained at least one stained myelin sheath (Table I). Therefore, we conclude that, as 

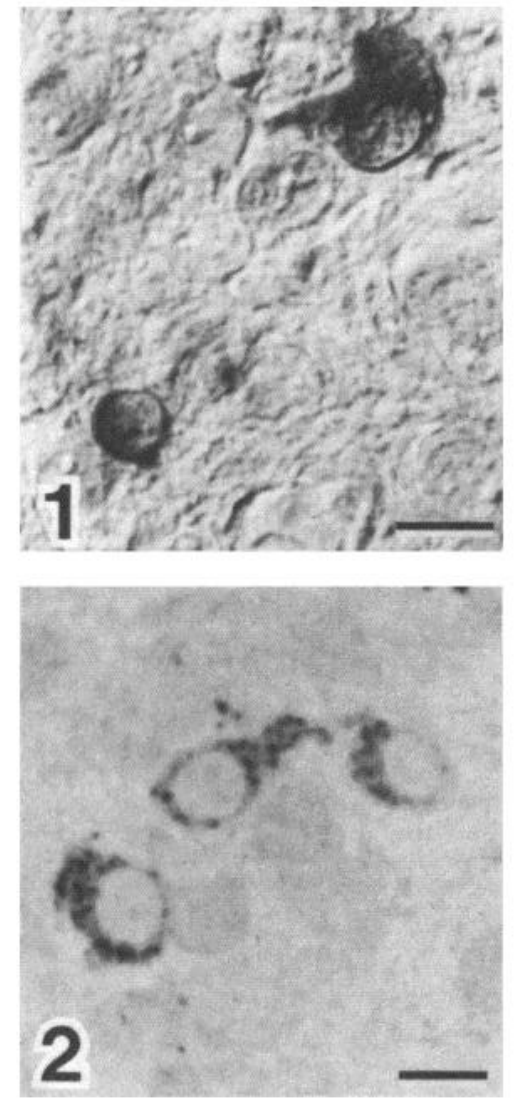
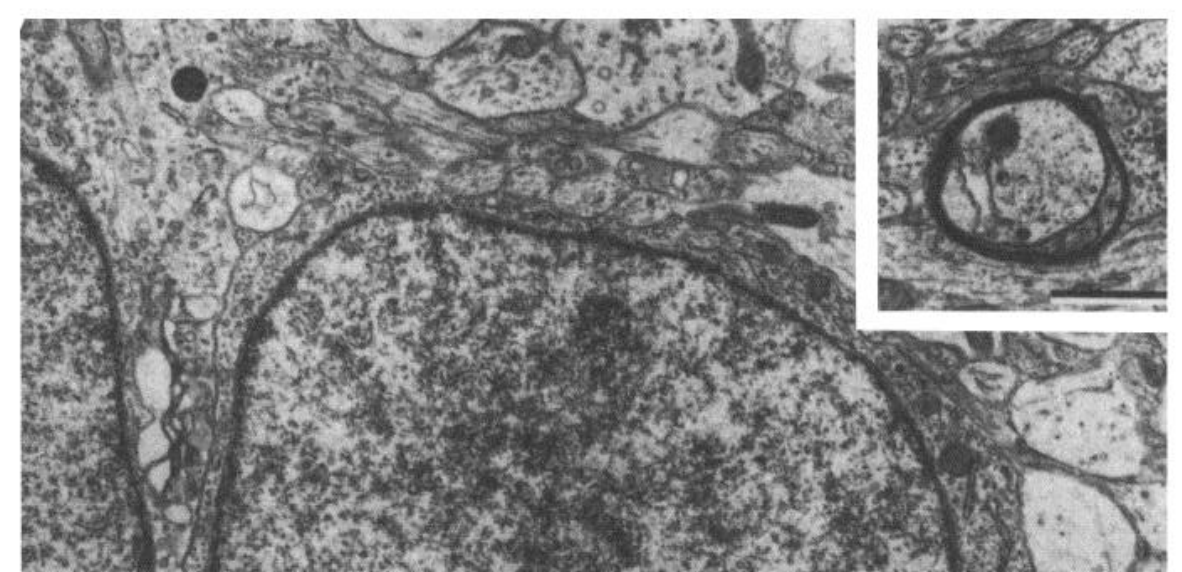

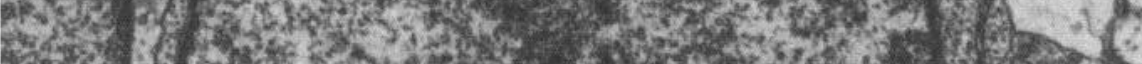

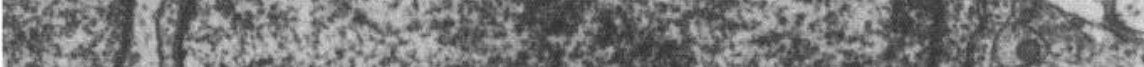

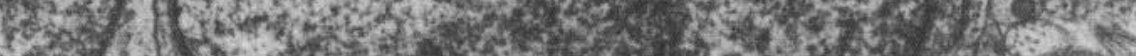

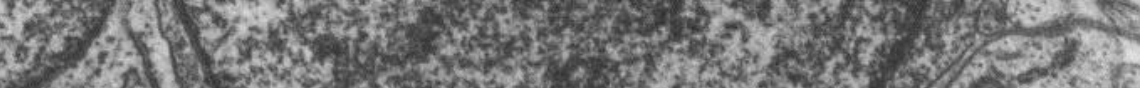

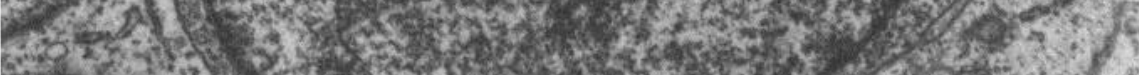

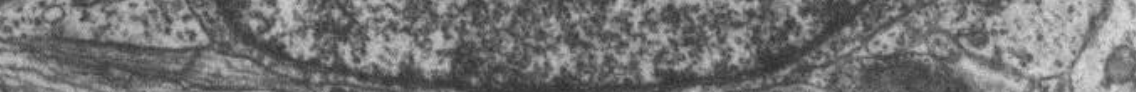

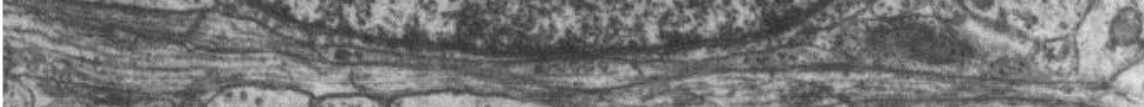

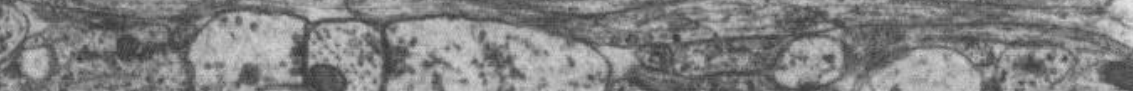

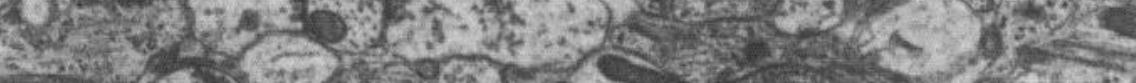

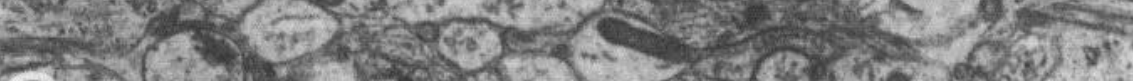
3.) rastin
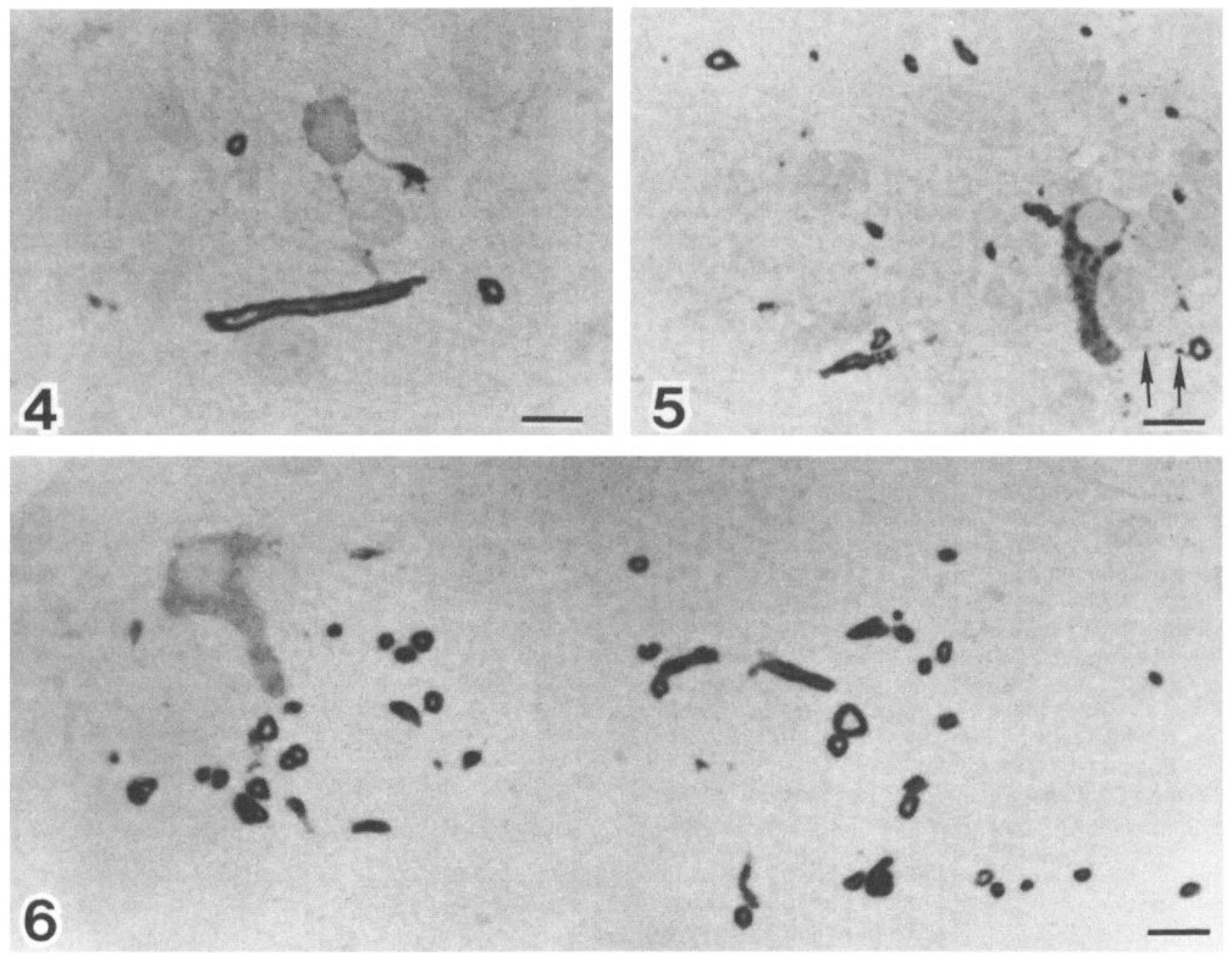
TABLE I

Percentage of aggregate sections that contain oligodendrocytes and myelin sheaths immunostained by MBP and MAG antisera

\begin{tabular}{ccrrrr}
\hline \multirow{2}{*}{ Days In Vitro } & \multicolumn{2}{c}{ Oligodendrocytes } & & \multicolumn{2}{c}{ Myelin } \\
\cline { 2 - 3 } \cline { 5 - 6 } \cline { 5 - 6 } & \multicolumn{1}{c}{ MBP } & \multicolumn{1}{c}{ MAG } & & MBP & \multicolumn{1}{c}{ MAG } \\
\hline 15 & $32.6(113)^{a}$ & $1.6(171)$ & & $10.2(113)$ & $0(171)$ \\
20 & $64.0(282)$ & $29.0(245)$ & & $49.0(282)$ & $19.2(245)$ \\
25 & $100(357)$ & $100(381)$ & & $100(357)$ & $100(381)$ \\
30 & $100(286)$ & $100(264)$ & & $100(286)$ & $100(264)$ \\
\hline
\end{tabular}

${ }^{a}$ Number of $20-\mu \mathrm{m}$ sections analyzed.

in vivo (Sternberger et al., 1978b), MBP appears in oligodendroglia before myelin sheath formation starts. Oligodendrocytes and a few axons surrounded by newly formed myelin sheaths were present in electron micrographs of 15-day aggregates (Fig. 3). Oligodendrocytes contained large nuclei which were surrounded by a thin rim of cytoplasm that contained free ribosomes, mitochondria, and occasional profiles of rough endoplasmic reticulum. Prominent Golgi membranes and microtubules were not present in these morphologically immature cells. Axons surrounded by newly formed myelin membranes were found rarely in thin sections of 15-day aggregates. When present, these axons had a diameter of $1 \mu \mathrm{m}$ or less and were surrounded by 2 to 4 loosely or partially compacted myelin lamellae (Fig. 3). In MAGtreated Vibratome sections from 15-day aggregates, myelin sheaths were not stained, and only $2.0 \%$ of these sections contained MAG-positive oligodendrocytes.

As the cells within the aggregates continued to mature, the number of oligodendrocytes and myelin sheaths stained by MBP and MAG antisera increased. Sixty-four percent of the MBP-treated Vibratome sections from 20 day aggregates contained stained oligodendrocytes and $49 \%$ contained stained myelin sheaths. Many of the MBP-stained oligodendrocytes had processes which were often continuous with myelin sheaths. When Vibratome sections from 20-day aggregates were treated with MAG antiserum, $29 \%$ of the sections contained MAG-positive oligodendroglia and 19\% contained MAG-stained myelin. In 1- $\mu \mathrm{m}$-thick Epon sections, MAG antiserum produced a diffuse staining of oligodendrocyte cytoplasm (Fig. 2). In addition, intense granular staining of cytoplasm and processes occurred. Oligodendrocytes and myelinated ax-
TABLE II

$M B P, M A G$, and CNP levels in aggregate homogenates All values are the mean of three separate samples measured in duplicate.

\begin{tabular}{|c|c|c|c|}
\hline Days In Vitro & MBP & MAG & CNP \\
\hline & \multicolumn{2}{|c|}{ ng/ug protein } & $\mu \mathrm{mol} / \mathrm{hr} / \mathrm{mg}$ protein \\
\hline 15 & $\mathrm{ND}^{a}$ & $\mathrm{ND}$ & ND \\
\hline 20 & ND & ND & 11 \\
\hline 25 & 2.25 & 0.30 & 91 \\
\hline 30 & 4.87 & 1.76 & 192 \\
\hline
\end{tabular}

${ }^{a} \mathrm{ND}$, not detected.

ons were more abundant in electron micrographs of 20 . day aggregates. Many of these cells displayed a relatively immature morphology, while other cells had begun to differentiate morphologically as determined by an increase in cytoplasmic volume and cytoplasmic organelles and the presence of microtubules. Myelinated axons were present in most sections. Some axons had just begun to be myelinated and others were surrounded by 6 to 8 compact lamellae.

All of the MBP- and MAG-treated Vibratome sections from 25- and 30-day aggregates contained at least one stained oligodendrocyte and myelin sheath. Immunostained cells in the 25- and 30-day aggregates had processes which were continuous with stained myelin sheaths (Figs. 4 and 5). A large mass of cytoplasm often extended from one pole of these immunostained oligodendrocytes. The number of immunostained myelin sheaths per aggregate section increased between 25 (Fig. 4) and 30 days (Fig. 6), but the number of MBP- and MAG-stained oligodendrocytes appeared to remain constant. This increase in the number of myelin sheaths demonstrated immunocytochemically paralleled increases in the levels of MBP and MAG (Table II).

In electron micrographs of 25- and 30-day aggregates, morphologically mature oligodendrocytes and numerous myelinated axons were present (Figs. 7 and 8). Myelinated axons were 0.8 to $2.6 \mu \mathrm{m}$ in diameter and were surrounded by compact myelin sheaths with 4 to 22 spirally wrapped lamellae. The periodicity of the myelin lamellae was identical to that found in vivo. Myelin segments terminated in paranodal loops, forming heminodes of Ranvier. Complete nodes of Ranvier were not identified in electron micrographs and were seen only

Figure 1. Twenty-micrometer-thick Vibratome sections from a 15-day aggregate stained with a 1:500 dilution of MBP antiserum. Two densely stained oligodendrocytes are present. Differential interference contrast optics were used. Scale bar, 10 $\mu \mathrm{m}$; magnification $\times 950$.

Figure 2. One-micrometer-thick Epon section of a 20-day aggregate stained with a 1:250 dilution of MAG antiserum. Intense particulate staining occurs throughout the cytoplasm of three oligodendrocytes. Bright-field optics were used. Scale bar, $10 \mu \mathrm{m}$; magnification $\times 850$.

Figure 3. Electron micrograph of a 15-day aggregate; oligodendrocyte has an undifferentiated (immature) fine structure. Newly formed myelin sheaths are present in 15-day aggregates (inset). Scale bar, $1 \mu \mathrm{m}$; magnification $\times 10,500$; inset magnification $\times$ 12,000 .

Figures 4 and 5. One-micrometer-thick Epon section from a 25-day aggregate stained with a 1:500 dilution of MBP (Fig. 4) and a 1:250 dilution of MAG (Fig. 5). MBP antiserum diffusely stains oligodendrocyte cytoplasm. MBP-stained processes can be traced from the oligodendrocyte to two stained myelin sheaths. MAG antiserum produces intense particulate staining of oligodendrocyte cytoplasm. A partially stained process (arrows) can be traced from this oligodendrocyte to a MAG-stained myelin sheath. Bright-field optics were used. Scale bars, $10 \mu \mathrm{m}$; magnification $\times 850$.

Figure 6. One-micrometer-thick Epon section from a 30-day aggregate stained with a 1:500 dilution of MBP antiserum. A stained oligodendrocyte and many MBP-stained myelin sheaths are present. Bright-field optics were used. Scale bar, $10 \mu \mathrm{m}$; magnification $\times 850$. 

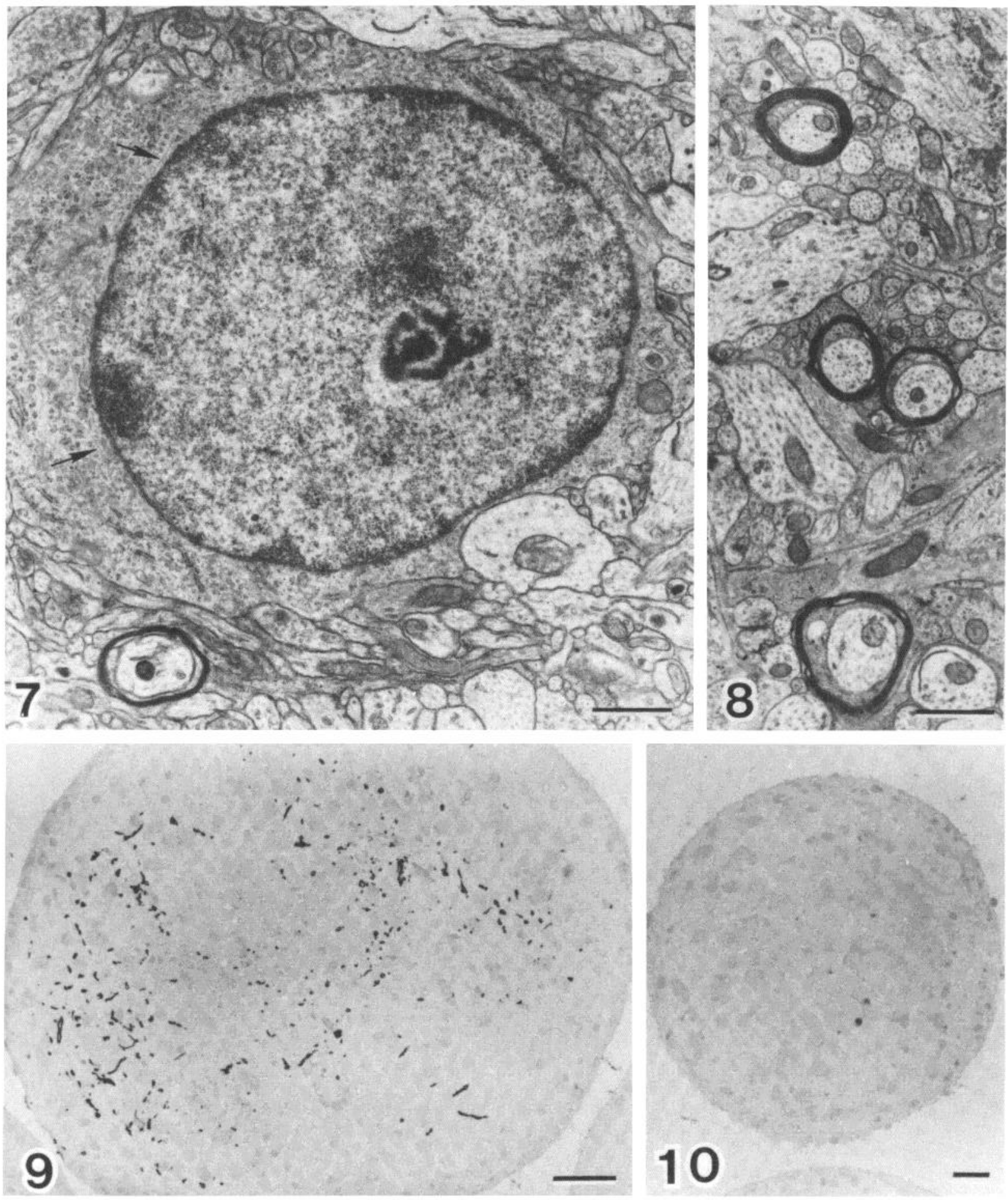

Figure 7. Electron micrograph of an oligodendrocyte from a 30-day aggregate. The cytoplasm contains microtubules (arrows), free ribosomes, rough endoplasmic reticulum, and Golgi membranes. Scale bar, $1 \mu \mathrm{m}$; magnification $\times 12,800$.

Figure 8. Electron micrograph of a 30-day aggregate. Four myelinated axons are present. Myelinated axons have a small diameter and the inner tongue processes are prominent. Scale bar, $1 \mu \mathrm{m}$; magnification $\times 13,000$.

Figure 9. One-micrometer-thick Epon section of a 30-day aggregate stained with a 1:500 dilution of MBP antiserum. Myelin sheaths are distributed throughout the aggregate. Bright-field optics were used. Scale bar, $50 \mu \mathrm{m} ;$ magnification $\times 200$.

Figure 10. Control 1- $\mu$ m-thick Epon section of a 30-day aggregate stained with MBP-absorbed antiserum. No staining of oligodendrocytes or myelin is observed. Bright-field optics were used. Scale bar, $50 \mu \mathrm{m}$; magnification $\times 120$. 
rarely in immunostained sections. Redundant myelin sheaths occurred occasionally within the aggregates. These aberrant myelin membranes usually meandered within the neuropil or, in rare cases, surrounded or were next to oligodendroglial perikarya.

In immunostained 1- $\mu \mathrm{m}$ Epon sections of 30-day aggregates, myelinated axons often appeared in clusters or groups which were distributed randomly throughout the aggregates (Fig. 9). Myelin sheaths were sectioned longitudinally, obliquely, or in cross-section. MBP and MAG staining was restricted to oligodendrocyte cytoplasm and myelin membranes in all sections studied. Neurons, astrocytes, oligodendrocyte nuclei, and axons were unstained. The specificity of the immunostaining procedure was demonstrated by incubating sections from 30-day aggregates with either preimmune sera or with antigenabsorbed MBP and MAG antisera. These sections were unstained (Fig. 10).

Biochemistry. Two myelin-specific proteins, MBP and MAG, were measured by radioimmunoassay in aggregate homogenates at $15,20,25$, and 30 days in vitro (Table II). The myelin-related enzyme, $2^{\prime}, 3^{\prime}$-cyclic nucleotide $3^{\prime}$ phosphodiesterase (CNP), was assayed also. MBP and MAG were not detected by radioimmunoassay in the 15and 20-day aggregate homogenates, whereas very low CNP activity was present in the 20-day homogenate. The levels of each of these components increased substantially in the aggregates at 25 and 30 days in vitro (Table II). Myelin was isolated from 30-day aggregate homogenates, and the yield of myelin protein was $0.9 \%$ of the total protein in the initial homogenate. The specific activity of CNP in the purified myelin was $1,095 \mu \mathrm{mol} /$

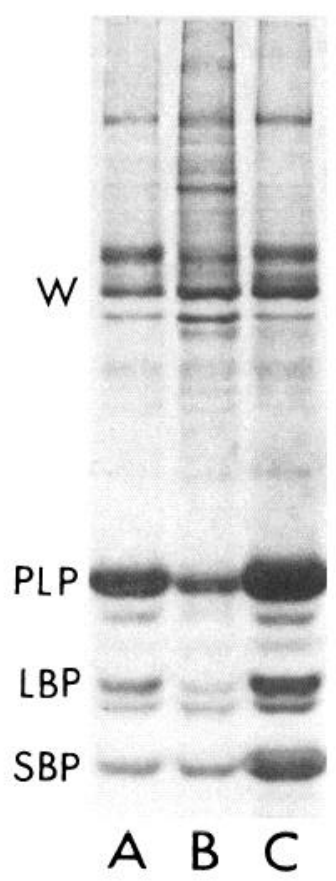

Figure 11. SDS slab gel (12.5\%) comparing myelin proteins from 14-day-old rats $(A)$, 30-day aggregates $(B)$, and an adult rat $(C)$. Wolfgram $(W)$, proteolipid $(P L P)$, large myelin basic protein $(L M P)$, and small myelin basic protein $(S B P)$ are present. $\mathrm{hr} / \mathrm{mg}$ of protein, a value similar to that in myelin purified from whole rat brain. Figure 11 shows an SDS slab gel comparing the protein composition of the myelin isolated from the 30-day aggregates with that of myelin isolated from 14-day and adult rat brains. All of the major rat myelin proteins were present in the aggregate myelin, but it contained a greater proportion of high molecular weight proteins than the myelin from the normal brains.

\section{Discussion}

Biochemical and morphological aspects of myelin formation have been investigated in aggregating cell cultures (Schmidt, 1975; Bourre et al., 1979; Sheppard et al., 1978; Matthieu et al., 1978, 1979, 1980; Trapp et al., 1979a; Trapp and Richelson, 1980), but no systematic multidisciplinary analysis of myelination has been reported. The purpose of this study was to correlate immunocytochemical, electron microscopic, and biochemical parameters of myelination in aggregating cultures derived from a single pool of fetal rat brains and maintained under identical culture conditions. Although our results support earlier studies describing many similarities in the process of myelination between aggregating cultures and the intact animal, a fundamental difference exists. Neurons and glia do not form a consistent histiotypic organization within the aggregating cultures used in this study. Myelination does not occur in an orderly manner along well defined tracts in which an intimate relationship between developing axons and oligodendrocytes exists. Myelin sheaths remain relatively thin and do not have a large number of compact lamellae. This lack of tract configuration may prolong oligodendrocyte differentiation and axon-glia interactions and may prove advantageous for studying early stages of myelination in vitro.

In previous publications describing the morphological differentiation of aggregating cell cultures (Matthieu et al., 1978; Trapp et al., 1979a), ultrastructural evidence of myelination was not detected at 19 days in vitro. By increasing the concentration of glucose in the culture medium to $600 \mathrm{mg} \%$ in the present study, myelination was detected at 15 days in vitro, and the amount of myelin in mature aggregates ( $\geq 25$ days in vitro) was greatly increased. Identical concentrations of glucose $(600 \mathrm{mg} \%)$ have similar effects on myelination in explant cultures (Bornstein, 1973).

The first evidence of myelination in aggregating cell cultures is the appearance of MBP in oligodendrocyte cytoplasm. As myelination proceeds, MBP-stained pro cesses appear, and these processes eventually surround axons and ferm compact myelin sheaths. Although MAG appears (in oligodendrocyte cytoplasm and myelin sheaths) somewhat later than MBP, it first appears in oligodendrocyte cytoplasm and then in oligodendrocyte processes which eventually become continuous with myelin sheaths. Therefore, three stages of myelinogenesis can be detected immunocytochemically: (1) the synthesis of MBP and MAG in perinuclear regions of oligodendrocytes, (2) the occurrence of these proteins in oligodendrocyte processes, and (3) the presence of these proteins in myelin sheaths. This pattern of myelinogenesis and 
MBP and MAG staining are similar to those found in normal developing rat brain (Sternberger et al., 1978a, b, 1979; Webster et al., 1981).

As in normal rat brain (Sternberger et al., 1978a, b), aggregate oligodendrocytes began to myelinate at different times. Oligodendrocytes displayed a more differentiated fine structure at progressively older in vitro ages. Oligodendrocyte cytoplasmic volume increases, microtubules appear, and rough endoplasmic reticulum and Golgi membranes become more abundant. Glycogen and fibrils, abundant constituents of astrocyte cytoplasm, are not present in oligodendrocytes. As these cells mature ultrastructurally, the number of MBP- and MAG-stained oligodendrocyte processes and myelin sheaths increase. Based on an analysis of electron micrographs from 15day aggregates, oligodendrocytes which contained only MBP staining within their cytoplasm are relatively undifferentiated. In electron micrographs of 20-day aggregates, some oligodendrocytes contained microtubules and an increase in cytoplasmic volume and organelles. Oligodendrocytes and myelin sheaths were more abundant in electron micrographs from 25-day aggregates. Oligodendrocytes contained microtubules and prominent Golgi complex membranes. The number of MBP- and MAG-stained oligodendrocytes increased between 20 and 25 days and almost all of these cells had stained processes which were continuous with stained myelin sheaths. In electron micrographs from 30-day aggregates, oligodendrocytes were morphologically mature. Most myelin sheaths in 30-day aggregates consisted of 10 to $15 \mathrm{com}$ pact lamellae and the diameter of myelinated axons averaged between 1 and $2 \mu \mathrm{m}$. In vivo, the number of myelin lamellae surrounding a CNS axon increases with axonal diameter (Bernstein, 1966). The number of myelin lamellae surrounding aggregate axons may be limited by the failure of axons to attain diameters greater than 2 $\mu \mathrm{m}$. Although the number of MBP- and MAG-stained myelin sheaths increased between 25 and 30 days, no appreciable increase in the number of stained oligodendrocytes occurred.

When aggregate oligodendrocytes were stained with MAG antiserum, intense granular staining of the cytoplasm and processes occurred. Similar granular staining has been described in vivo (Sternberger et al., 1979; Webster et al., 1981). A similar pattern of staining has been described in areas of Golgi complex membranes of myelinating Schwann cells stained by $P_{0}$ antiserum (Trapp et al., 1981a). Po and MAG are both integral membrane glycoproteins. If their sites of synthesis are similar, the granular MAG staining in perikarya may represent oligodendrocyte Golgi complex membranes. As compact myelin sheaths grow in thickness in vivo, only periaxonal portions are stained by MAG antiserum (Sternberger et al., 1979). The periaxonal localization of MAG was not evident in aggregate myelin sheaths, as they do not contain enough compact lamellae to visualize unstained portions with the light microscope. Ultrastructural localization may determine if MAG is restricted to periaxonal portions of aggregate myelin sheaths. Comparison of MBP- and MAG-stained 20- $\mu \mathrm{m}$-thick Vibratome sections from 15- and 20-day aggregates demon- strated that a greater percentage of oligodendrocytes and myelin sheaths were stained by MBP antiserum. Based on quantitation of the number of MBP- and MAGstained oligodendrocytes in Vibratome sections of the developing rat anterior commissure, MBP appears in oligodendrocytes prior to MAG in vivo (Sternberger et al., 1978a, 1979). These results suggest that MBP can be detected immunocytochemically in oligodendrocytes and myelin sheaths prior to MAG. Electron microscopic localization of MBP and MAG during initial contacts of oligodendrocytes and axons may clarify any specific role MBP or MAG may serve in the initiation of myelination.

Our results clearly demonstrate that the immunostaining techniques used are very sensitive, highly specific methods for detecting MBP and MAG in oligodendrocytes and myelin membranes. Immunostaining was more sensitive than biochemical methods for detecting MBP and MAG, but it is limited by the inability to quantitate the amount of protein present. Radioimmunoassay for MBP and MAG revealed significant levels in aggregate cultures at 25 days in vitro, the age at which $100 \%$ of the Vibratome sections examined contained at least one oligodendrocyte and myelin sheath that was stained for MBP and MAG. The levels of MAG and CNP in the 30day aggregates were $66 \%$ and $56 \%$, respectively, of their levels in whole adult rat brain. By contrast, the level of basic protein in the 30-day aggregates was only $8 \%$ of that in adult rat brain. The very low level of basic protein is probably due to the fact that myelin sheaths which form are short and relatively thin and do not have a large number of compact lamellae. However, the periaxonal myelin and other oligodendrocyte-derived membranes which are enriched in MAG and CNP (Quarles, 1979; Sternberger et al., 1979) probably are formed in amounts more closely approaching adult levels. Measurements of these components during normal in vivo development of rat brain also have revealed that MAG and CNP approach adult levels at an earlier age than basic protein (Sprinkle et al., 1978; Johnson et al., 1980). The yield and composition of the myelin isolated from the 30-day aggregates suggest that it is biochemically immature. The yield was comparable to that obtained from an 18-day rat brain, and the large content of high molecular weight proteins is typical for immature myelin (Morell et al., 1972). Similarly, Matthieu et al. (1979) have concluded that myelin formed in aggregating cultures is biochemically immature.

In conclusion, the morphological, immunocytochemical, and biochemical results indicate that aggregate cultures provide a useful in vitro system for studying the early stages of myelinogenesis.

\section{References}

Bernstein, J. J. (1966) Relationship of cortico-spinal tract growth to age and body weight in the rat. J. Comp. Neurol. 127: 207-218.

Bornstein, M. B. (1973) Organotypic mammalian central and peripheral nerve tissue. In Tissue Culture Methods and Applications, P. F. Kruze and M. K. Patterson, eds., pp. 86-92, Academic Press, New York. 
Bourre, J. M., P. Honegger, O. Daudu, and J. M. Matthieu (1979) The lipid composition of rat brain aggregating cell cultures during development. Neurosci. Lett. 11: 275-278.

Cohen, S. R., G. M. McKhann, and M. Guarnieri (1975) A radioimmunoassay for myelin basic protein and its use for quantitative measuremęnts. J. Neurochem. 25: 371-376.

Garber, B. B. (1977) Cell aggregation and recognition in the self-assembly of brain tissues. In Cell, Tissue, and Organ Culture in Neurobiology, S. Fedoroff and L. Hertz, eds., pp. 515-538, Academic Press, New York.

Honegger, P., and E. Richelson (1976) Biochemical differentiation of mechanically dissociated mammalian brain in aggregating cell cultures. Brain Res. 109: 335-354.

Honegger, P., and E. Richelson (1977) Biochemical differentiation of aggregating cell cultures of different fetal brain regions. Brain Res. 133: 329-339.

Honegger, P., and E. Richelson (1979) Neurotransmitter synthesis, storage and release by aggregating cell cultures of rat brain. Brain Res. 162: 89-101.

Johnson, D., R. H. Quarles, and R. O. Brady (1980) A radioimmunoassay for the myelin associated glycoprotein. Fed. Proc. 39: 1831.

Kurihara, T., and Y. Tsukada (1967) The regional and subcellular distribution of $2^{\prime}, 3^{\prime}$-cyclic nucleotide $3^{\prime}$-phosphohydrolase in the central nervous system. J. Neurochem. 14: $1167-1174$

Laemmli, U. K. (1970) Cleavage of structural protein during the assembly of the head of bacteriophage 'T4. Nature 227: $680-685$.

Lowry, O. H., N. J. Rosebrough, A. L. Farr, and R. J. Randall (1951) Protein measurement with Folin-phenol reagent. J. Biol. Chem. 193: 265-275.

Matthieu, J. M., P. Honegger, B. D. Trapp, S. R. Cohen, and $\mathrm{H}$. deF. Webster (1978) Myelination in rat brain aggregating cell cultures. Neuroscience 3: 565-572.

Matthieu, J. M., P. Honegger, P. Favrod, E. Gautier, and M. Dolivo (1979) Biochemical characterization of a myelin fraction isolated from rat brain aggregating cell cultures. J. Neurochem. 32: 869-881.

Matthieu, J. M., P. Honegger, P. Favrod, J. P. Poduslo, E. Costantino-Ceccarini, and R. Krstic (1980) Myelination and demyelination in aggregating cultures of rat brain cells. In Tissue Culture in Neurobiology, E. Giacobini, A. Vernadakis, and A. Shahar, eds., pp. 441-459, Raven Press, New York.

Morell, P., S. Greenfield, E. Costantino-Ceccarini, and H. Wisniewski (1972) Changes in the protein composition of mouse brain myelin during development. J. Neurochem. 19: 2545-2554.

Norton, W. T., and S. E. Poduslo (1973) Myelination in rat brain: Method of myelin isolation. J. Neurochem. 21: 749757.

Quarles, R. H. (1979) Glycoproteins in myelin and myelinrelated membranes. In Complex Carbohydrates of the Nervous System, R. U. Margolis and R. K. Margolis, eds., pp. 209-233, Plenum Press, New York.

Quarles, R. H., and J. L. Everly (1977) Glycopeptide fractions prepared from purified central and peripheral rat myelin. Biochim. Biophys. Acta 466: 176-186.

Quarles, R. H., D. Johnson, R. O. Brady, and N. H. Sternberger (1981) Preparation and characterization of antisera to the myelin-associated glycoprotein. Neurochem. Res. 6: 1109-1121.
Schmidt, G. L. (1975) Development of biochemical activities associated with myelination in chick brain aggregate cultures. Brain Res. 87: 110-113.

Seeds, N. W. (1973) Differentiation of aggregating brain cell cultures. In Tissue Cultures of the Nervous System, G. Sato, ed., pp. 35-53, Plenum Press, New York.

Sheppard, J. R., D. Brus, and J. M. Wehner (1978) Brain reaggregate cultures: Biochemical evidence for myelin membrane synthesis. J. Neurobiol. 9: 309-315.

Sprinkle, T. J., M. E. Yaruba, and G. M. McKhann (1978) Activity of $2^{\prime}, 3^{\prime}$-cyclic nucleotide $3^{\prime}$-phosphodiesterase in regions of rat brain during development: Quantitative relationship to myelin basic protein. J. Neurochem. 30: 309-314.

Sternberger, L. A., P. H. Hardy, J. J. Cuculus, and H. G. Meyer (1970) The unlabeled antibody enzyme method of immunohistochemistry. Preparation and properties of soluble antigen-antibody complex (horseradish peroxidase-antihorseradish peroxidase) and its use in identification of spirochetes. J. Histochem. Cytochem. 18: 315-333.

Sternberger, N. H., Y. Itoyama, M. W. Kies, and H. deF. Webster (1978a) Myelin basic protein demonstrated immunocytochemically in oligodendroglia prior to myelin sheath formation. Proc. Natl. Acad. Sci. U. S. A. 75: 2521-2524.

Sternberger, N. H., Y. Itoyama, M. W. Kies, and H. deF Webster (1978b) Immunocytochemical method to identify basic protein in myelin-forming oligodendrocytes of newborn rat CNS. J. Neurocytol. 7: 251-263.

Sternberger, N. H., R. H. Quarles, Y. Itoyama, and H. deF. Webster (1979) Myelin-associated glycoprotein demonstrated immunocytochemically in myelin and myelin-forming cells of developing rats. Proc. Natl. Acad. Sci. U. S. A. 76: 1510-1514.

Trapp, B. D., and E. Richelson (1980) Usefulness for neurotoxicology of rotation-mediated aggregating cell cultures. In Experimental and Clinical Neurotoxicology, P. S. Spencer and H. H. Schaumburg, eds., pp. 803-819, Williams and Wilkins, Baltimore.

Trapp, B. D., P. Honegger, E. Richelson, and H. deF. Webster (1979a) Morphological differentiation of mechanically dissociated fetal rat brain in aggregating cell cultures. Brain Res. 150: $117-130$.

Trapp, B. D., L. J. McIntrye, R. H. Quarles, N. H. Sternberger, and $H$. deF. Webster (1979b) Immunocytochemical localization of rat peripheral nervous system myelin proteins: P2 protein is not a component of all peripheral nervous system myelin sheaths. Proc. Natl. Acad. Sci. U. S. A. 76: 3552-3556.

Trapp, B. D., L. J. McIntyre, H. deF. Webster, and M. Murray (1979c) Myelination in rotation mediated aggregating cell cultures. Soc. Neurosci. Abstr. 5: 2562.

Trapp, B. D., Y. Itoyama, N. H. Sternberger, R. H. Quarles, and $H$. deF. Webster (1981a) Immunocytochemical localization of Po protein in Golgi complex membranes and myelin of developing rat Schwann cells. J. Cell Biol. 90: 1-6.

Trapp, B. D., P. J. Marangos, and H. deF. Webster (1981b) Immunocytochemical localization and developmental profile of neuron specific enolase (NSE) and non-neuronal enolase (NNE) in aggregating cell cultures of fetal rat brain. Brain Res. 220: 121-130.

Webster, H. deF., B. D. Trapp, N. H. Sternberger, and R. H. Quarles (1981) Myelin-forming glial cells: Morphological and immunocytochemical observations. In Development in the Nervous System, D. R. Garrod and I. D. Feldman, eds., pp. 265-288, Cambridge University Press, Cambridge, England. 